Uribe, D., Acevedo, M., Rojas, O., Yojcom, D. (2020) Comparación de hallazgos matemáticos en grupos culturales diferenciados mediante el modelo metodológico MOC - ETNO. Revista Latinoamericana de Etnomatemáticas, 13(2), 45-65 DOI: 10.22267/relatem.20132.60

Artículo recibido el 12 de abril de 2020. Aceptado para publicación el 14 de julio del 2020

\title{
Comparación de hallazgos matemáticos en grupos culturales diferenciados mediante el modelo metodológico MOC - ETNO
}

\section{Comparison of mathematical findings in differentiated cultural groups using the MOC - ETNO methodological model}

\author{
David Uribe Suarez ${ }^{1}$ \\ Myriam Acevedo ${ }^{2}$ \\ Osvaldo Rojas ${ }^{3}$ \\ Domingo Yojcom ${ }^{4}$
}

\begin{abstract}
Resumen
Este artículo presenta el modelo metodológico comparativo con enfoque etnomatemático (MOC-ETNO), compuesto de cuatros fases: descripción, interpretación, comparación y acciones, para identificar y contrastar las prácticas ancestrales de las culturas Wayuu y Arhuaca respecto al saber y al hacer con patrones de medida. Este estudio es parte de una investigación doctoral, cuyo objetivo fue diseñar un modelo, sustentado y fundamentado teóricamente en cada una de las dimensiones del programa de etnomatemática, con un principal interés en las actividades matemáticas universales propuestas por Bishop y en el uso del método comparativo. La metodología de investigación se ubica en el paradigma de investigación cualitativo - hermenéutico, con un enfoque relativista y de tipo etnográfico.
\end{abstract}

Palabras clave: Etnomatemática; Prácticas Culturales; Actividades Matemáticas; Modelo Metodológico.

\begin{abstract}
This article presents the comparative methodological model with an ethnomathematical approach (MOCETNO), consisting of four phases: description, interpretation, comparison and actions, to identify and contrast the ancient practices of the Wayuu and Arhuaca cultures with respect to knowing and doing with patterns measure. This study is part of a doctoral research, the objective of which was to design a model, supported and theoretically based on each of the dimensions of the ethnomathematics program, with a main interest in the universal mathematical activities proposed by Bishop and in the use of the comparative method. The research methodology is located in the qualitative-hermeneutical research paradigm, with a relativistic and ethnographic approach.

Key words: Ethnomathematics; Cultural Practices; Mathematical Activities; Methodological Model.

1 Ingeniero Industrial, Especialista en Pedagogía y Docencia. Doctorando en Educación Matemática, Universidad Antonio Nariño, Riohacha, Colombia. Email: daviduribe246@uan.edu.co

2 Licenciado en matemáticas, Magister en Matemática, Universidad Nacional de Colombia, Bogotá, Colombia. Email: mmacevedoc@unal.edu.co

${ }^{3}$ Licenciado en Educación matemática, Doctor en ciencias pedagógicas, Universidad Antonio Nariño, Bogotá, Colombia. Email: orojasv69@uan.edu.co

4. Profesorado en Matemáticas y Física, Maestría en Educación/Currículo especialidad en Matemáticas, Doctor en Ciencias con Especialidad en Matemática Educativa, Ciudad de Guatemala, Guatemala. Email: centrocientificogt@gmail.com
\end{abstract}




\section{INTRODUCCIÓN}

Las concepciones acerca de la naturaleza de la educación han evolucionado, dejando de lado los énfasis y enfoques tradicionales respecto al proceso de enseñanza-aprendizaje centrado en el contenido. Este proceso se ha fundamentado exclusivamente en el saber disciplinar formal, a nivel mundial. En la actualidad se reconoce como primordial incorporar el conocimiento propio de las diferentes culturas, los contextos y entornos próximos al estudiante, al proceso educativo, para contribuir a mejorar sus conocimientos. Asumir la diversidad cultural en los campos socioeconómicos, políticos, lingüísticos, educacionales, en particular el relacionado con la educación matemática, entre otros, constituye un reto para las entidades de educación en los diferentes países en la actualidad. Este proceso se puede llevar a cabo considerando las nuevas tendencias renovadoras de la educación matemática, específicamente la etnomatemática, la cual permite transformar y modificar el proceso de enseñanza aprendizaje de las matemáticas en los diferentes niveles educativos.

Desde esta perspectiva, Bishop plantea que "el conocimiento matemático se ha construido socialmente y que a través de su desarrollo han existido y existen, diferentes manifestaciones y prácticas del pensamiento matemático en diversos entornos culturales, evidenciándose en ellas actividades matemáticas universales” (Bishop, 2005, p. 36). Estas actividades matemáticas universales (AMU) poseen ciertas diferencias con algunas de las actividades que se trabajan formalmente en el sistema escolar.

En el mismo sentido, D’Ambrosio afirma que "el aporte interdisciplinar de los antropólogos, los historiadores de las culturas y los matemáticos como paso fundamental al reconocimiento de que diferentes modos de pensamientos, puede llevar a diferentes formas de matemáticas, o ideas matemáticas" (D’Ambrosio, 1985, p. 44). A través de estas ideas se puede reconocer, que algunas de las dificultades del proceso de enseñanza y aprendizaje de las matemáticas en contextos indígenas, desconocen o no involucran sus prácticas matemáticas ancestrales en el entorno escolar.

Retomando planteamientos anteriores, la UNESCO afirma que:

"las diferentes formas de diversidad, diversidades socio-económicas, culturales, lingüísticas y de genero se ha constituido un verdadero reto en la calidad de la 
Uribe, D., Acevedo, M., Rojas, O., Yojcom, D. (2020) Comparación de hallazgos matemáticos en grupos culturales diferenciados mediante el modelo metodológico MOC - ETNO. Revista Latinoamericana de Etnomatemáticas, 13(2), 45-65 DOI: 10.22267/relatem.20132.60

educación matemática, y que alrededor de los desafíos que ha generado la diversidad cultural y las implicaciones al proceso de enseñanza aprendizaje de las matemáticas, el campo de la Etnomatemática ha contribuido sustancialmente a crear conciencia para que la calidad de la educación matemática mejore continuamente” (UNESCO, 2012, p. 39).

Teniendo en cuenta la perspectiva mencionada respecto a la posibilidad de caracterizar prácticas matemáticas propias en diferentes contextos y culturas, en el desarrollo de la investigación, una vez diseñado el modelo metodológico, se aplicó para comparar prácticas de la cultura wayuu en las comunidades de Anaralito y Arroyo, para identificar en ellas el uso de patrones de medida autóctonos y comparar con las prácticas de la cultura Arhuaca. En la fundamentación de esta esta investigación se revisaron antecedentes relacionados con los diferentes enfoques y desarrollos de la Etnomatemática, en particular aquellos que reconocen y describen actividades matemáticas universales desde la cotidianidad de grupos culturales diferenciados.

Algunos de los antecedentes pertinentes que fueron consultados se describen sucintamente a continuación. El estudio de Valverde y Espinoza (2012) es desarrollado en el contexto indígena ulwas de la comunidad Karawala de Nicaragua, donde identifican prácticas matemáticas socioculturales, que representan saberes etnomatemáticos ancestrales. Ávila (2017) desarrolló una investigación con la población indígena cabécar en Costa Rica, en la que encontró actividades métricas diferentes a las occidentales para la construcción de la base del rancho para la vivienda.

Trujillo, Miranda y De la Hoz (2018) desarrollaron una investigación en la comunidad Arhuaca de la Sierra Nevada de Santa Marta, con el propósito de identificar medidas autóctonas utilizadas por esta comunidad en un contexto escolar, comunitario y socio cultural.

En el informe del sexto Congreso Internacional de Etnomatemática, se encuentran dos referencias respecto a las actividades matemáticas universales. García, Mosquera y Escobar (2018) describe una investigación sobre las medidas en la práctica sociocultural agrícola del cultivo del arroz y Gavarrete, Martinez y Chavarría (2018) indagó sobre las medidas tradicionales en pueblos indígenas de Costa Rica. 
La investigación del presente artículo se sustentó teóricamente en el Programa de Etnomatemática, en lo que respecta a las dimensiones propuestas por D’Ambrosio (2000), a las actividades matemáticas universales planteadas por Bishop (1999), y se apoyó en el estudio del método comparativo con sus respectivas etapas. Desde esta perspectiva, se aborda la etnomatemática como un programa de investigación científica, que pretende describir e interpretar las diversas formas de conocer de los distintos grupos culturales en su esfuerzo por sobrevivir y transcender.

En el programa de investigación de etnomatemática se identifican seis dimensiones que estructuran su abordaje: conceptual, histórica, cognitiva, epistemológica, política y educativa (D’Ambrosio, 2000), en las cuales se ubican los trabajos desarrollados en el área. La investigación que se describe en este artículo enfatiza la dimensión cognitiva, pues tiene en cuenta que en todo grupo cultural existen algunas ideas matemáticas que son visibilizadas en la cotidianidad del grupo, es decir, que la vida cotidiana de cualquier cultura está impregnada de matemáticas.

Existen seis actividades matemáticas universales que todas las culturas manifiestan en algún grado, estas son: contar, localizar, medir, diseñar, jugar y explicar (Bishop, 1999). Esta investigación enfatiza la medición, así mismo el trabajo se fundamentó en la caracterización de las fases del método comparativo propuestas por Bereday (1964): descripción, interpretación, yuxtaposición, comparación y una fase que agrega Caballero, Manso, Matarranz y Valle (2016), denominada etapa prospectiva.

En el modelo metodológico que se propone se unen las fases de yuxtaposición y comparación, por ser la primera implícita en la segunda. La yuxtaposición como acción única y estática de colocar solo los fenómenos en paralelo para su respectiva comparación, por lo que puede quedar como actividad de la fase de comparación.

\section{METODOLOGÍA}

La investigación desarrollada se ubica dentro del paradigma de investigación cualitativo hermenéutico, con un enfoque relativista y de tipo etnográfico para la aplicación de dicho modelo metodológico. La etnografía trata de describir o interpretar algunos fenómenos humanos, a menudo en palabras propias de los informantes seleccionados, igualmente Balcázar, González, López, Gurrola y Moysén (2015), plantean que el investigador intenta 
Uribe, D., Acevedo, M., Rojas, O., Yojcom, D. (2020) Comparación de hallazgos matemáticos en grupos culturales diferenciados mediante el modelo metodológico MOC - ETNO. Revista Latinoamericana de Etnomatemáticas, 13(2), 45-65 DOI: 10.22267/relatem.20132.60

observar cómo los miembros del grupo ven, sienten, experimentan y construyen cognitivamente su mundo.

La metodología que sugiere D’Ambrosio (2000) para investigar en Etnomatemática se fundamenta en la observación y el análisis de las prácticas de comunidades y grupos diferenciados, para observar qué hacen y por qué realizan lo que hacen.

\section{RESULTADOS}

Como herramienta para llevar a cabo y alcanzar los resultados de comparación de hallazgos matemáticos, en grupos culturalmente diferenciados, se utilizó la perspectiva educativa del Programa de Etnomatemática y para ello se construyó un Modelo Metodológico Comparativo con Enfoque Etnomatemático. Este modelo metodológico es aplicable para la identificación y comprensión de actividades matemáticas universales en contextos de grupos culturalmente diferenciados y minoritarios tales como: indígenas, afrodescendientes, raizal colombiano, grupos de profesionales, grupos de personas con oficios comunes, entre otros.

Además, MOC-ETNO es ubicado en dos enfoques dentro de la etnomatemática. Primero en el enfoque de la Matemática y Etnomatemática planteada por Fuentes (2014), donde afirma que la matemática es algo aceptado por la etnomatemática, es decir, no se rechaza la matemática disciplinar. En segundo lugar, se tiene el enfoque de estudios etnomatemáticos, que aluden a grupos culturalmente diferenciados, categorizados por Rosa, Orey y Gavarrete mediante la siguiente descripción para:

“otorgar significado a los contenidos matemáticos, personalizarlos y contextualizarlos en el marco social, cultural, geográfico y temporal, mediante problemas para ser implementados en el aula de clases" ( Rosa, Orey y Gavarrete, 2017, p. 76).

El modelo tiene una estructura que consta de dos secciones, una vertical y una horizontal, la vertical está compuesta por cuatro fases: descripción, interpretación, yuxtaposición/comparación y acciones, (Figura 1). 


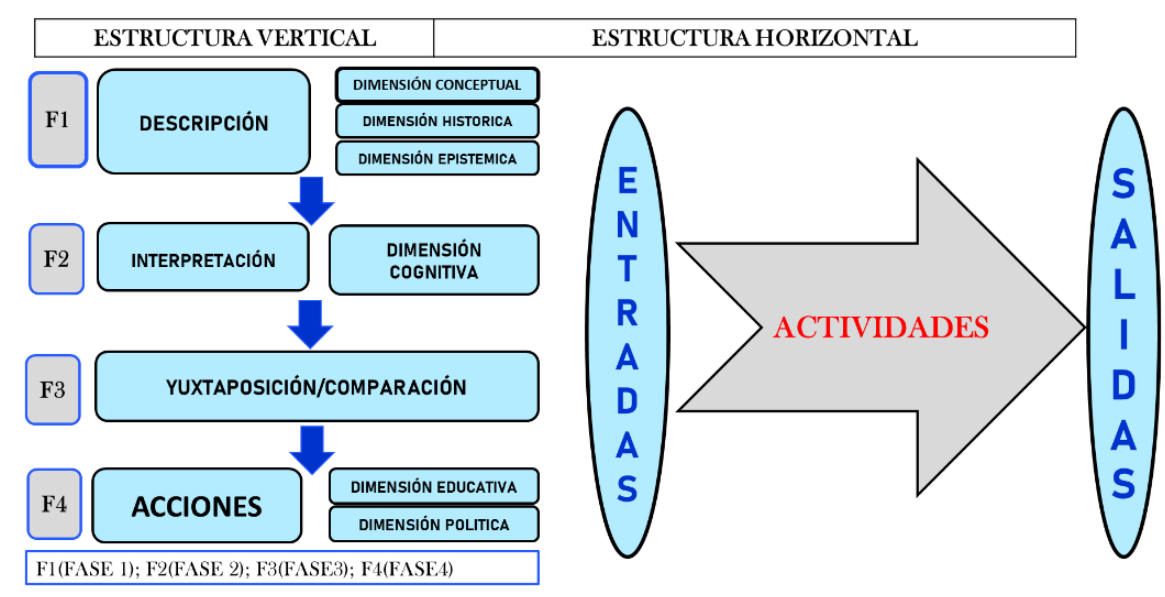

Figura 1. Representación general del modelo metodológico. Construcción de los autores

Las fases están soportadas con el método comparativo según los trabajos de Santamaría (1983) y Bereday (1964), además cada fase, tiene una interrelación específica con alguna de las dimensiones de la Etnomatemática como programa de investigación. Están asociadas de la siguiente manera, la fase uno se asocia a las dimensiones conceptual, histórica y epistemológica; la fase dos a la dimensión cognitiva y la fase cuatro a las dimensiones política y educativa. Se procede a explicar cada fase vertical del modelo metodológico con su respectiva sección horizontal.

\subsection{Fase uno. Descripción}

En la fase de descripción (Figura 2), según el método comparativo y lo afirmado por Santamaría (1983), no solo se dan los primeros pasos del proceso, sino que se intenta adquirir un conocimiento robusto y lo más completo posible de lo que se quiere comparar. Para el enfoque de este modelo metodológico el tipo de conocimiento al que se hace referencia es el conocimiento matemático construido socialmente en grupos culturalmente diferenciados, que intenta visibilizar algunas actividades matemáticas universales desde las prácticas culturales cotidianas, definidas por Bishop (1999) y/o algunas ideas matemáticas descritas por D’Ambrosio (2000) desde la dimensión cognitiva de la etnomatemática. 
Uribe, D., Acevedo, M., Rojas, O., Yojcom, D. (2020) Comparación de hallazgos matemáticos en grupos culturales diferenciados mediante el modelo metodológico MOC - ETNO. Revista Latinoamericana de Etnomatemáticas, 13(2), 45-65 DOI: 10.22267/relatem.20132.60

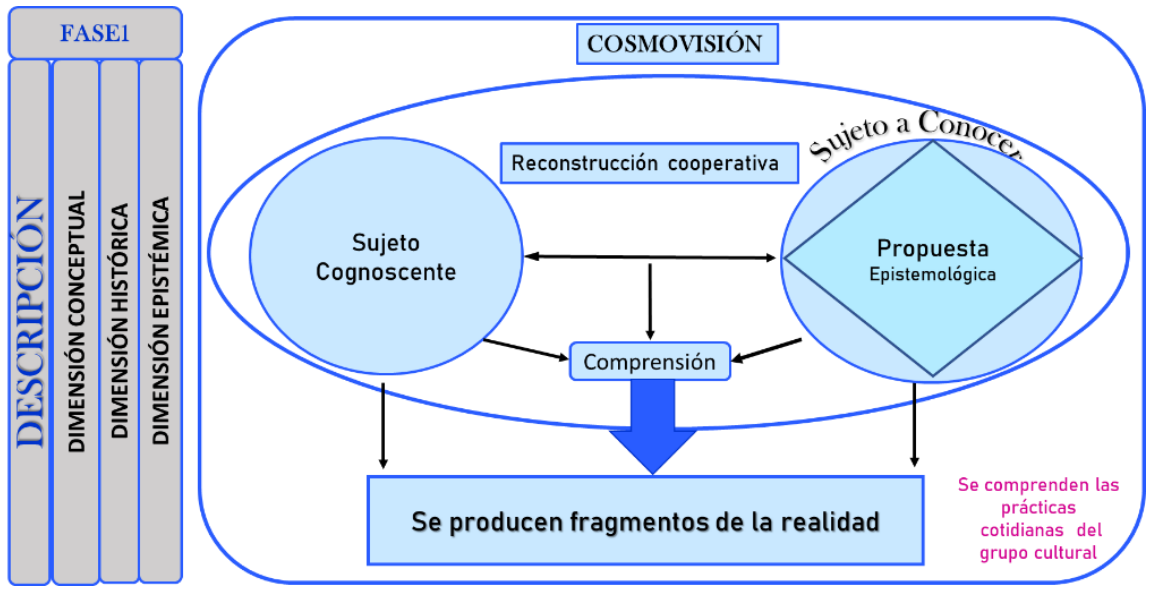

Figura 2. Fase uno: Descripción. Adaptación Luque (2010, p. 30)

A continuación, una breve explicación de los conceptos básicos presentados en la figura 2, relacionado a las dimensiones conceptual, histórica y epistemológica en conexión con la primera etapa de la descripción. Es importante para el investigador antes de emprender cualquier acción en esta fase, tener claro y definido los grupos culturales en los cuales quiere aplicar su comparación.

Al aplicar este modelo es posible para el investigador seleccionar grupos culturales, en los que se han realizado estudios previos, que han permitido visibilizar su pensamiento matemático desde sus prácticas cotidianas, pues con ellos, puede confrontar sus propios hallazgos o confirmar la información develada con un estudio empírico propio.

La dimensión conceptual en la descripción: la matemática, como el conocimiento en general, es una respuesta a los impulsos de supervivencia y de trascendencia (D’Ambrosio, 2000), para lo cual hay situaciones que implican la esencia de la existencia de la especie humana, logrando que la humanidad desde cada grupo social cree sus propias teorías y realicen prácticas que van soportando la construcción del conocimiento.

Acorde con esta postura es importante que el investigador a través de las vivencias, del relato de las prácticas cotidianas y de los conocimientos que permitan develar, describa, en cada grupo cultural, la manera cómo este ha enfrentado su supervivencia en el espacio y en el tiempo, y cómo ha transcendido hasta el tiempo presente.

La dimensión histórica en la descripción: la etnomatemática reconoce otras formas de pensamiento que siempre van en paralelo con manifestaciones de la cultura. Por lo tanto, la 
descripción bajo este enfoque histórico está relacionada con el abordaje de la propia historia de los grupos culturalmente diferenciados, que serán objeto de estudio. Es por ello, que una de las tareas en esta fase es describir la historia del grupo contada por ellos mismos, dejando que sus relatos muestren las manifestaciones de su pensamiento y permitan conocer más a fondo su realidad y la esencia de sus prácticas cotidianas.

La dimensión epistemológica en la descripción: esta dimensión está relacionada con los sistemas de conocimientos, desde como emergen, su organización estructural y social, su difusión y el retorno de estos conocimientos a los grupos culturales responsables de su producción. Esta característica permite trabajar esta dimensión con cualquier grupo cultural.

De la descripción anterior es posible inferir que las tres dimensiones están ligadas entre sí, y trabajan de manera teórica y práctica intentando entender el saber hacer matemático de los pueblos y culturas minoritarias. Con respecto a la sección horizontal de esta primera fase (descripción), se definen los elementos esenciales de entrada, se describe cada actividad y elementos del proceso, y se concluye especificando los resultados como las salidas de dicho proceso.

Elementos de entrada fase primera: generalmente los investigadores inician definiendo los grupos culturales que son objeto de estudio para la comparación y verifican si existe literatura o investigaciones referentes a las actividades matemáticas universales de los grupos a estudiar.

Es imprescindible gestionar la entrada a los grupos culturales, en este caso, una de las primeras acciones fue acudir a las autoridades tradicionales de las comunidades del Arroyo y Anaralito. Estas autoridades aprobaron la entrada a la comunidad y a la escuela para realizar un estudio etnográfico inicial y visibilizar las actividades matemáticas universales o para confrontar hallazgos de estudios preliminares o antecedentes.

Si el investigador no pertenece a la comunidad es necesario tener un intérprete. En el ejercicio de esta investigación se contó con un miembro de la comunidad para esta función. Además, cuando la lengua de la comunidad no es la misma del investigador, el intérprete puede ayudar a develar los significados de las prácticas, cosmovisión y cosmogonía de la comunidad. 
Uribe, D., Acevedo, M., Rojas, O., Yojcom, D. (2020) Comparación de hallazgos matemáticos en grupos culturales diferenciados mediante el modelo metodológico MOC - ETNO. Revista Latinoamericana de Etnomatemáticas, 13(2), 45-65 DOI: 10.22267/relatem.20132.60

Una vez definidos los elementos de entrada se procede a estructurar cada uno de forma particular. Esta fase muestra las actividades orientadas a comprender las prácticas cotidianas del grupo cultural y permite tener un fragmento claro de la realidad. Además, en muchos momentos de esta fase se despliegan indicios de actividades matemáticas universales que se sistematizan en la fase siguiente de interpretación.

Se soporta esta etapa inicial en la metodología de Luque (2010), sobre el elemento sujeto a conocer, adaptando el enfoque de construcción del conocimiento planteado por D’Ambrosio (2000), desde la dimensión epistemológica de la etnomatemática como una postura filosófica que debe asumir el investigador para intentar entender la episteme del sujeto a conocer. Estas ideas permiten además trabajar en cualquier grupo cultural y no solamente en grupos étnicos como refiere Luque.

Sujeto cognoscente: cumple la función de investigador, es el encargado de recolectar la información de campo de los grupos culturales. Si en los grupos culturales ya se han evidenciado actividades matemáticas universales en estudios previos, el investigador debe confrontar esos hallazgos, inmerso en la realidad, replicando su metodología y, además, si es posible, agregar nuevos hallazgos. El sujeto cognoscente no debe realizar interpretaciones de los fenómenos y cotidianidades que está observando desde sus propias representaciones porque la meta principal es apropiarse de la episteme del sujeto a conocer desde una perspectiva emic.

Así mismo, las interpretaciones que el investigador asuma deben ser dadas en conjunto con la episteme del sujeto conocido en el espacio de la reconstrucción cooperativa, que al final del proceso permita tener una comprensión de las prácticas culturales cotidianas y de los primeros indicios de actividades matemáticas universales impregnadas en ellas, $\mathrm{u}$ otras que se definan en la investigación. El proceso de interpretación es más enriquecedor cuando el sujeto cognoscente es parte de la cultura del sujeto a conocer, en este momento la reconstrucción cooperativa es más reflexiva y orientadora para los fines de la fase dos.

El sujeto a conocer: es la persona que pertenece al grupo cultural objeto de estudio, por tanto, es de vital importancia para el proceso de investigación en la fase de campo. Puede ser una o varias personas que conozcan muy bien la cultura, sus costumbres, mitos, tradiciones, prácticas cotidianas, entre otros. Es decir, debe ser reconocido por el grupo 
cultural como tal y además ser un digno representante de ella; en él está la mayor parte de la información para que, en conjunto con el sujeto cognoscente, permita construir fragmentos de la realidad fidedignos y replicables. Los resultados generados en la fase uno, el sujeto a conocer ya será sujeto conocido o parcialmente conocido.

Reconstrucción Cooperativa: este es el escenario donde el sujeto cognoscente, el sujeto a conocer y el intérprete trabajaron en conexión. Este proceso se realiza a partir de la episteme del sujeto a conocer con la fundamentación clara que debe tener el investigador del esquema de la propuesta epistemológica planteada por D’Ambrosio (2000), para entender el ciclo del conocimiento y para analizar el conocimiento matemático de las culturas periféricas. Igualmente, es el escenario real donde se trabaja la recolección de la información, aplicando una metodología desde el paradigma cualitativo, con el apoyo del método etnográfico.

En este proceso investigativo se aplican las fases necesarias para los intereses del grupo y de la investigación misma, y los instrumentos que requiere este tipo de investigación. Se sobreentiende el hecho de que el investigador es conocedor de este cuerpo de conocimiento para abordar estos tipos de estudios que conjugan lo antropológico con lo etnomatemático.

En el desarrollo de la investigación y la convivencia mutua entre los sujetos, el investigador debe procurar comprender la episteme del sujeto a conocer junto a la suya. Lógicamente esto es consecuencia de llevar a cabo las visitas de campo regular y repetidamente para que se registren detalladamente fragmentos de la realidad, de manera que al final del estudio, esos fragmentos puedan estar unidos y se genere una visión de la realidad lo más fidedigno posible desde las voces de sus informantes. El tiempo de prolongación y estadía en campo depende del investigador. Para este estudio se tiene un tiempo pre-investigativo de tres años en espacios intermitentes dentro de la comunidad para ir apropiándose de la cultura del grupo y ocho meses de trabajo de campo especifico.

Es claro que en este escenario y a través del proceso, el investigador avanza en la comprensión de detalles de la realidad en la cual está inmerso; al final como resultado se tiene una producción muy compleja de los fragmentos de la realidad de cada grupo cultural. En consecuencia, se tiene muy claro un inventario de prácticas culturales que potencialmente sirven para evidenciar actividades matemáticas universales y esto le permite 
Uribe, D., Acevedo, M., Rojas, O., Yojcom, D. (2020) Comparación de hallazgos matemáticos en grupos culturales diferenciados mediante el modelo metodológico MOC - ETNO. Revista Latinoamericana de Etnomatemáticas, 13(2), 45-65 DOI: 10.22267/relatem.20132.60

avanzar a la fase siguiente de este modelo metodológico. Para culminar esta primera fase se explica la propuesta epistemológica que resulta ser un agregado de la metodología planteada por Luque (2010) y que sirve de soporte para esta primera fase en la sección horizontal del modelo.

Propuesta epistemológica: la intención planteada en el programa de etnomatemática respecto a la epistemología, según D’Ambrosio (2000) no es proponer una epistemología más, sino es realizar un análisis crítico a la epistemología tradicional que orienta el cuerpo de conocimiento que ya está determinado. Este enfoque epistemológico tradicional no tiene en cuenta en su generación, organización intelectual, social y difusión, la retroalimentación con ese conocimiento a aquellos responsables de su producción, es decir, lo que en este modelo metodológico se les llama sujeto a conocer, representando a su comunidad como un informante clave y con un reconocimiento hecho por la misma comunidad. Por esta razón la propuesta epistemológica planteada por D’Ambrosio (2000), es adecuada para entender y analizar el conocimiento matemático de estas culturas diferenciadas o minoritarias. Tal propuesta se representa en el esquema de la Figura 3.

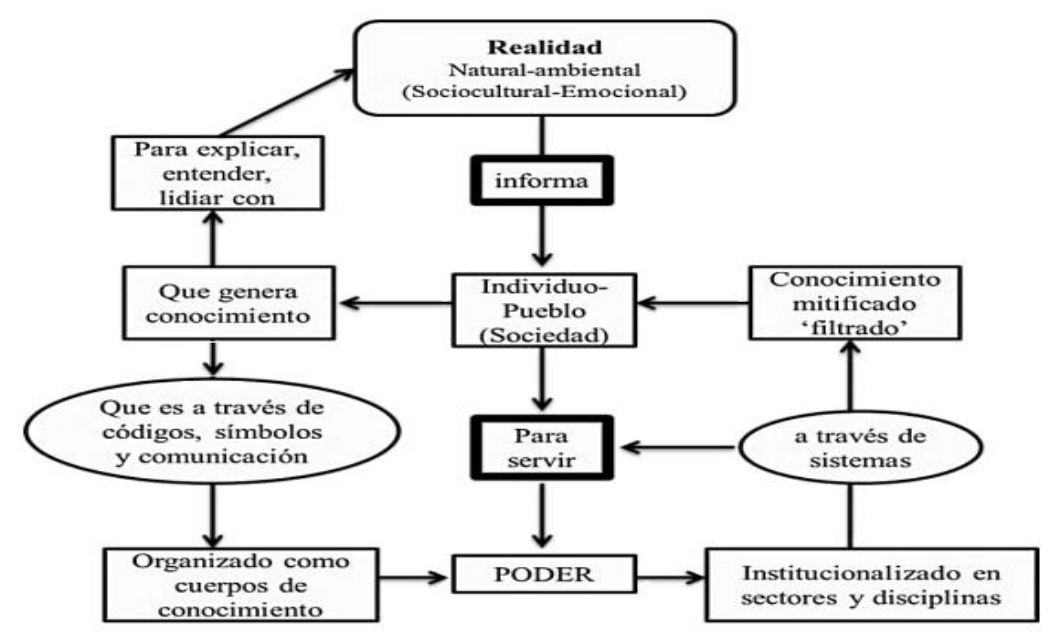

Figura 3. Esquema de la Propuesta Epistemológica. Adaptación D’Ambrosio 2000.

\subsection{Fase dos. Interpretación.}

En la fase de interpretación (Figura 4) el investigador seleccionó inicialmente una ruta, teniendo en cuenta el inventario de prácticas cotidianas culturales potenciales, con las cuales trabajó para realizar una comparación de acuerdo con los resultados de la fase anterior. Y teniendo en cuenta la elección, podrá asignar una codificación para la 
clasificación y organización de la información a recoger posteriormente.

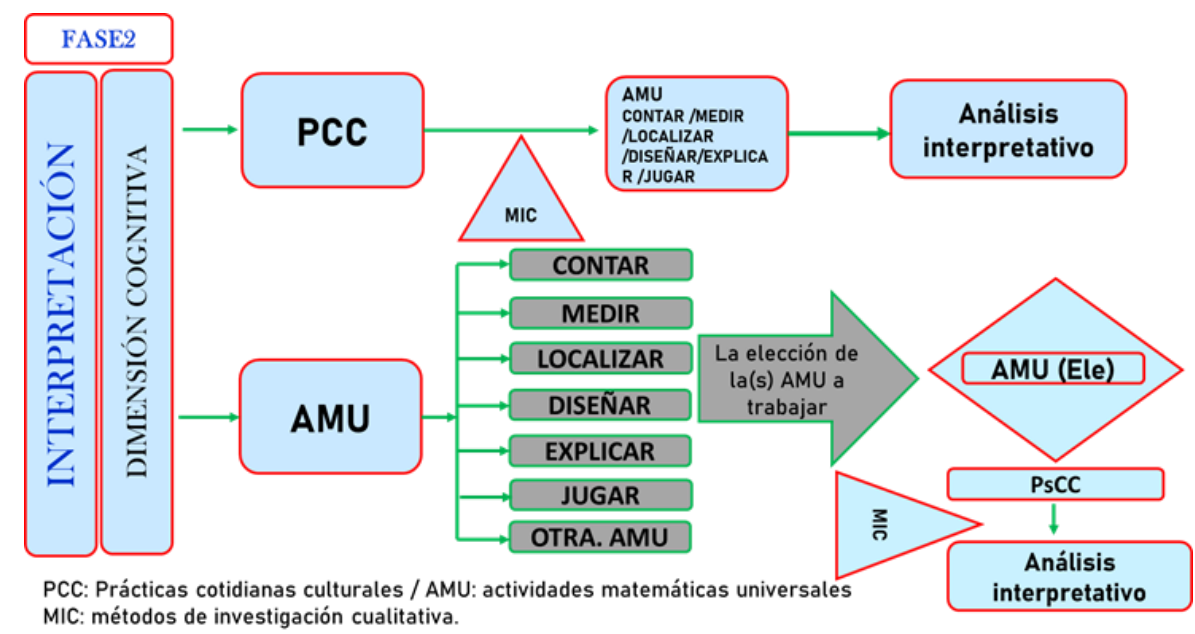

Figura 4. Fase dos: Interpretación. Construcción de los autores

La interpretación es un filtro de la etapa de descripción, por lo tanto, una vez definidas las prácticas cotidianas culturales, las actividades matemáticas universales, junto a los fragmentos de la realidad que se generaron, se debe volver a campo, esta vez solo focalizado en visibilizar las actividades matemáticas universales en las prácticas culturales cotidianas previamente definidas con la intención de realizar una explicación más contextualizada y detallada del fenómeno de estudio.

Dos rutas que el investigador podría elegir son: 1) seleccionar una o varias prácticas cotidianas culturales (Figura 5) y visibilizar mediante una interpretación desde las aplicaciones metodológicas del paradigma interpretativo, las seis actividades matemáticas universales u otras. 
Uribe, D., Acevedo, M., Rojas, O., Yojcom, D. (2020) Comparación de hallazgos matemáticos en grupos culturales diferenciados mediante el modelo metodológico MOC - ETNO. Revista Latinoamericana de Etnomatemáticas, 13(2), 45-65 DOI: 10.22267/relatem.20132.60

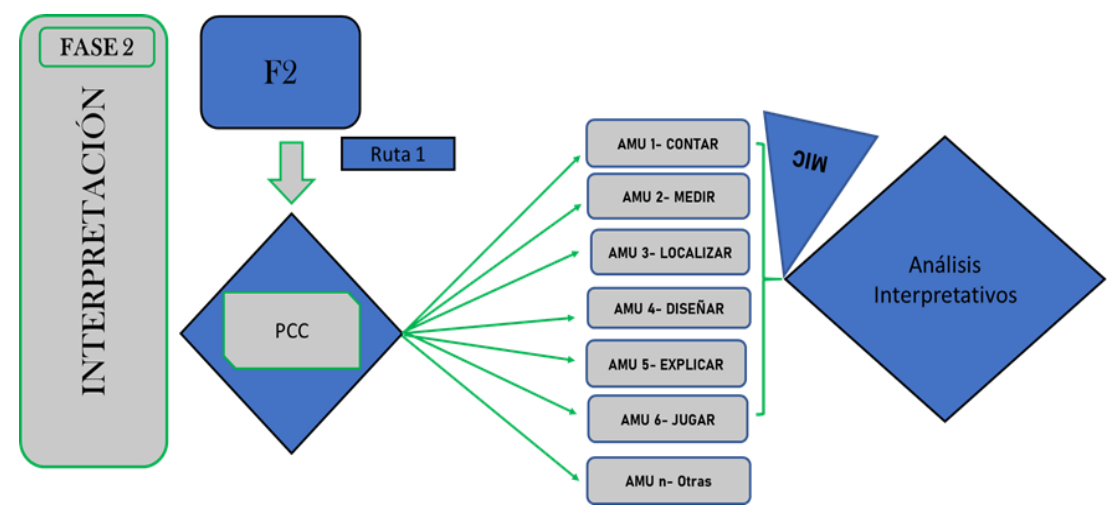

Figura 5. Ruta 1. Fase Interpretación. Construcción de los autores

O 2) elegir una o varias actividades matemáticas universales u otras representativas (Figura 6) del grupo cultural y tomar la mayor cantidad posible de prácticas cotidianas culturales para develar en ellas las actividades matemáticas universales elegidas, siendo esta la ruta elegida para esta investigación.

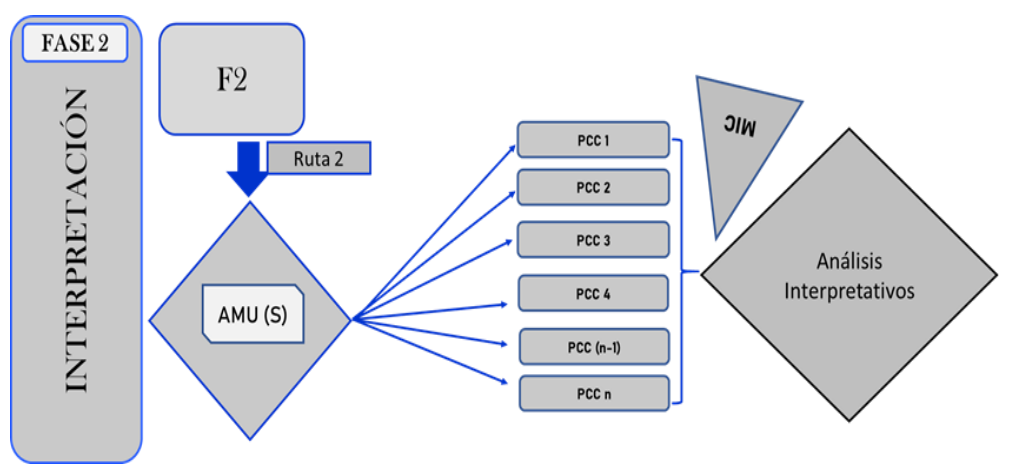

Figura 6. Ruta 2. Fase Interpretación. Construcción de los autores.

La cantidad de prácticas culturales cotidianas a elegir está a cargo del investigador, de acuerdo con los fines del estudio. Es importante resaltar que en la ruta uno la acción es, visibilizar dentro de muchas prácticas culturales una o varias AMU, sin dejar la posibilidad de que se puedan trabajar todas las actividades u otras pertinentes para el grupo.

Independiente de la ruta seleccionada se debe garantizar previamente los recursos que permitan iniciar, ejecutar y finalizar la investigación para que sus hallazgos sirvan como insumos tanto para la comparación y la construcción de propuestas didácticas que puedan ser llevadas al aula, considerando el contexto cultural del estudiante.

La dimensión cognitiva en la interpretación: el sujeto a estudiar es parte activa de un 
contexto cultural, él puede percibir y describir su realidad, manifestada en comportamientos y conocimientos que comparte con el grupo, esa realidad, es reflejada en las prácticas culturales cotidianas, en las que se usan las actividades matemáticas universales para resolver situaciones. Por lo anterior, es posible considerar al sujeto en la investigación como una fuente de conocimientos sobre los que el investigador pretende indagar y posibilitan una interpretación más fortalecida.

\subsection{Fase tres. Comparación.}

En esta fase, a través del método comparativo, se genera un diálogo intercultural entre dos o más grupos, donde se enriquece el análisis de sus prácticas y se evidencian semejanzas y diferencias entre las culturas. Esta comparación debe, entre otras cosas, conducir a la construcción de mejores propuestas pedagógicas en los grupos culturales objetos de la investigación. Esta fase se representa con las siguientes Figuras (7 y 8), la primera corresponde a la ruta uno y la segunda a la ruta dos (seleccionada para esta investigación), ya descritas en la fase anterior.

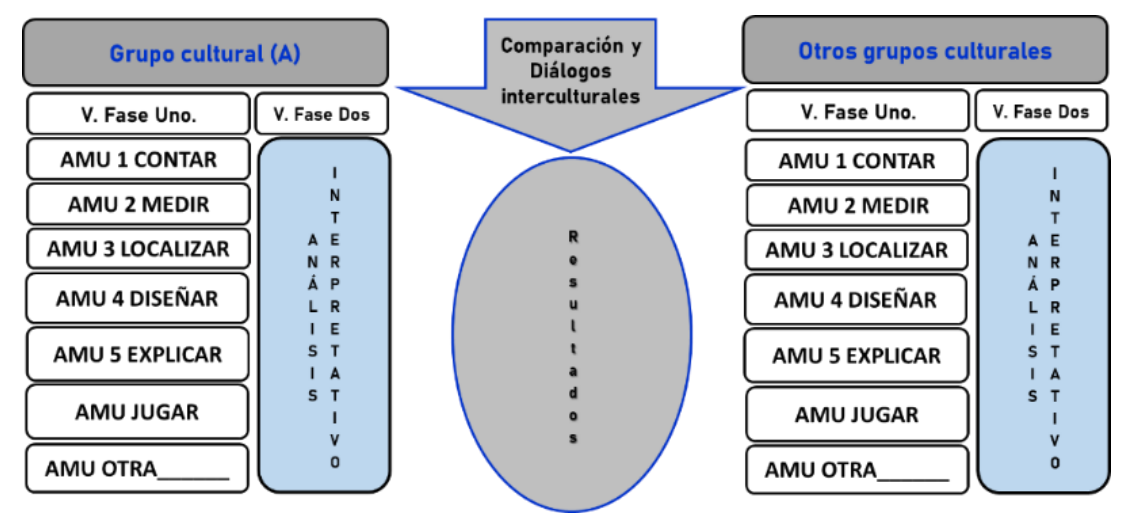

Figura 7. Fase comparación ruta 1. Construcción de los autores.

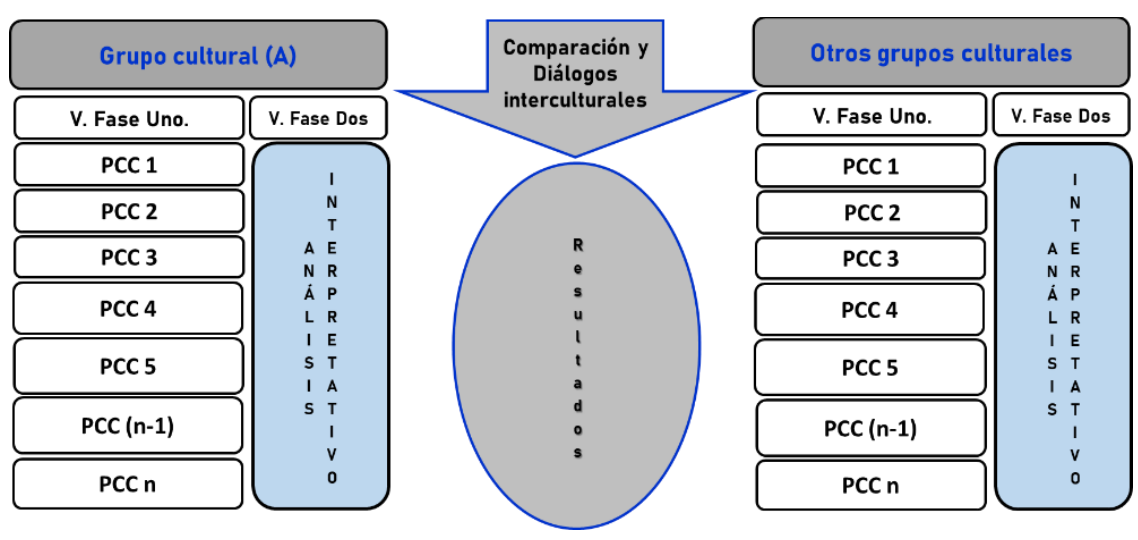


Uribe, D., Acevedo, M., Rojas, O., Yojcom, D. (2020) Comparación de hallazgos matemáticos en grupos culturales diferenciados mediante el modelo metodológico MOC - ETNO. Revista Latinoamericana de Etnomatemáticas, 13(2), 45-65 DOI: 10.22267/relatem.20132.60

Figura 8. Fase comparación ruta 2. Construcción de los autores.

En esta fase es necesario definir algunos aspectos que le dan relevancia a los resultados que arroje el análisis de carácter teórico, enriquecido por la experiencia de los estudios etnográficos realizados. Tales aspectos están relacionados con el método comparativo como herramienta científica para generar conocimiento.

Se observa en ambas gráficas, que ellas toman la información de entrada de fases anteriores. Faltan por definir entonces los aspectos que se tienen en cuenta para la comparación; esto se realiza a continuación.

Para la comparación se consideran: el conocimiento previo, el campo y la finalidad. El conocimiento previo de los grupos culturales que el investigador ha adquirido en las fases anteriores, le permite proceder con la estructura metódica de la comparación. Es importante la determinación previa del campo y las condiciones donde se va a aplicar el hecho comparativo, para precisar el empleo que se les dan a los resultados, es decir, la finalidad. La comparación con enfoque etnomatemático mediante el modelo metodológico MOCETNO puede proceder de una de las siguientes dos formas:

a) Estableciendo las características de transformación de un fenómeno, situación o práctica hasta lo que actualmente el grupo manifiesta.

b) Estableciendo relaciones entre las características de un fenómeno, situación o práctica entre dos o varios contextos.

Es claro que estas dos formas de proceder en la comparación son imprescindibles en la investigación científica, pues dan soporte al investigador acerca de los resultados que se obtienen. En la comparación se observa el comportamiento de unos fenómenos, situaciones o prácticas y además se trabaja con algunos constructos ya prestablecidos o realidades ya conocidas para poder descifrar al interior de ellas situaciones que a simple vista no surgen. Por ello, la comparación con enfoque etnomatemático permite el conocimiento de actividades matemáticas universales en prácticas culturales cotidianas de grupos diferenciados, en su esencia, señalando diferencias y semejanzas, pero con opciones de generar mejoras en diferentes escenarios educativos.

\subsection{Fase cuatro. Acciones.}


En esta última fase del modelo metodológico es importante que del diálogo intercultural teórico-práctico, que se genera de la comparación de actividades matemáticas universales en prácticas culturales cotidianas de los grupos y de los estudios etnográficos, resulten acciones o propuestas didácticas relativas al proceso de enseñanza aprendizaje de algunos conceptos y procedimientos matemáticos acordes con el contexto cultural y étnico. Esta fase se relaciona con las dimensiones política y educativa de la etnomatemática.

Esta fase comprende los elementos internos de estas dimensiones, que se muestran en la Figura 9: contenidos matemáticos, propuestas pedagógicas y la triada, conocimiento, profesor y estudiante en un contexto.

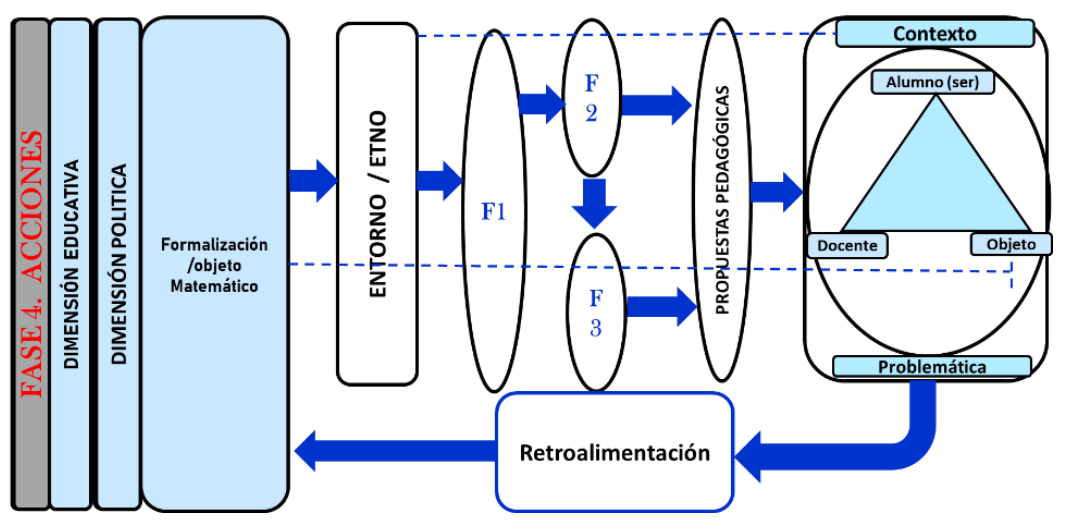

Figura 9. Fase de Acciones. Construcción de los autores.

La dimensión política, que aparece mencionada en el diagrama anterior, no se considera como una propuesta de negación de las políticas educativas oficiales del Ministerio de Educación Nacional (MEN), dado que esta institución prevé atención educativa para los grupos que integran la nacionalidad, con estrategias pedagógicas acordes con su cultura, su lengua, sus tradiciones y sus fueros propios y autóctonos. En esta dimensión se pretende fortalecer los procesos de enseñanza y aprendizaje, teniendo en cuenta la diversidad, pero en ningún momento, desconocer el conocimiento disciplinar formal, sino enriquecer este conocimiento teniendo en cuenta el conocimiento ancestral y cultural.

Entre las múltiples ventajas de esta dimensión, se pueden mencionar, el fortalecimiento de las raíces, la recuperación de la historia propia de los pueblos, el configurar la relación de lenguas y, además, dar utilidad a sus propios sistemas de explicación y a su forma de ver el mundo. Se pretende que los diálogos interculturales se den a un nivel horizontal, respetando, aceptando y construyendo sin jerarquizar el conocimiento según procedencia 
Uribe, D., Acevedo, M., Rojas, O., Yojcom, D. (2020) Comparación de hallazgos matemáticos en grupos culturales diferenciados mediante el modelo metodológico MOC - ETNO. Revista Latinoamericana de Etnomatemáticas, 13(2), 45-65 DOI: 10.22267/relatem.20132.60

cultural.

Para referirse a la dimensión educativa, se hace necesario retomar aquí la afirmación de D’Ambrosio (2000), en lo que respecta a la etnomatemática, ella no implica el rechazo de la matemática académica, pero es necesario que se afiancen valores en la humanidad como la ética, respeto, solidaridad y cooperación, apoyando los retos que han surgido en materia de etnoeducación. El modelo metodológico implementado en esta investigación adopta las posturas planteada por Bishop y D’Ambrosio intentado describir e interpretar en prácticas culturales la utilización de actividades matemáticas universales de grupos diferenciados, con la premisa de que existen varias matemáticas.

Con respecto a lo anterior se trata de tender un puente entre la matemática concebida como disciplina y los hallazgos de actividades matemáticas que visibilizan sus prácticas culturales cotidianas. Por lo tanto, desde esta dimensión y como resultado de esta fase, se diseñaron propuestas pedagógicas para el proceso de enseñanza aprendizaje de las matemáticas en cada grupo de manera particular.

Objetos matemáticos: Existe un conocimiento matemático disciplinar, formal establecido, este conocimiento, se estructura y transforma para ser abordado en una secuencia formal en los diferentes niveles educativos. Sin necesidad de profundizar en un debate de cuál debe ser un objeto matemático mediado, es necesario que el contenido escolar que se lleve al aula, como resultado de esta fase, esté íntimamente ligado al contexto cultural.

La aplicación de esta metodología va a permitir, al sujeto, profundizar en el conocimiento de las prácticas del grupo, desde su cosmogonía y cosmovisión, lo que le facilitará establecer ese puente entre el contenido matemático disciplinar y lo que el grupo en su cotidianidad hace como propio, concretándolo con una educación matemática adecuada para el contexto. En muchas ocasiones florecerán modos de explicaciones, inferencias, algoritmos, etc., que difieren a las formas de hacer de la matemática académica y que deberán ser prevalecientes para ese grupo en particular dentro de esas propuestas que se generen.

Propuestas Pedagógicas: Este elemento está relacionado con el diseño y la potencialización de propuestas para ser llevadas al aula, será una dinámica de un largo 
proceso para que cada grupo social articule su proceso de enseñanza aprendizaje de las matemáticas con una postura Etnomatemática. No se afirma que se evidenciaran transformaciones inmediatas en las aulas, pero con la construcción de ellas se plantea otra ruta de cambio para que el docente pueda abordar las matemáticas en contextos sociales minoritarios y/o diferenciados.

Ellas deben ser diseñadas acorde a la relación entre el objeto matemático y la forma como se evidencia en la práctica cultural del grupo, donde el estudiante podrá apropiarse de sus raíces culturales, pero también paralelamente va trabajando por comprender y apropiarse de un conocimiento matemático formal/universal y construido por una comunidad.

Comunidad de Aprendizaje: Es el escenario de aplicación de propuestas pedagógicas, donde el docente debe ser pieza fundamental para que los estudiantes reciban esos conocimientos y saberes adecuadamente. En la triada entre el saber, el estudiante, el docente, y adicionalmente con el contexto, se pueden solucionar problemas de aprendizaje identificados en este escenario, con la aplicación continua de propuestas contextualizadas, su implementación conlleva siempre a aspectos de retroalimentación logrando afinar más y más los contenidos que se estén llevando a esta comunidad.

El proceso de validación del modelo metodológico comienza tomando los criterios consensuados que se derivan de la aplicación del Método Delphi ${ }^{1}$, los cuales enriquecen esta propuesta. El modelo metodológico propuesto se validó con la puesta en práctica de cada una de sus fases. En su concreción práctica el modelo permite: describir e interpretar actividades matemáticas universales en prácticas cotidianas de grupos culturales, la comparación y generación de diálogos interculturales desde la diversidad y del diseño de actividades didácticas llevadas al aula. Los resultados aportados por cada una de estas fases del proceso investigativo, permitió concluir con la validación del modelo metodológico.

\section{CONCLUSIONES}

El modelo metodológico MOC- ETNO es una herramienta que puede ser aplicada por la comunidad de investigadores en etnomatemática y docentes de grupos culturales

\footnotetext{
${ }^{1}$ Para orientaciones del uso del Método Delphi en investigaciones educativas puede consultar el siguiente enlace. https://www.redalyc.org/pdf/706/70653466002.pdf
} 
Uribe, D., Acevedo, M., Rojas, O., Yojcom, D. (2020) Comparación de hallazgos matemáticos en grupos culturales diferenciados mediante el modelo metodológico MOC - ETNO. Revista Latinoamericana de Etnomatemáticas, 13(2), 45-65 DOI: 10.22267/relatem.20132.60

minoritarios, para iniciar, continuar y fortalecer diálogos interculturales. Además, la aplicación de este modelo también permitirá fortalecer la enseñanza de las matemáticas de las instituciones etno-educativas, diseñando propuestas de aula que acerquen al estudiante a su contexto, generando aprendizaje significativo de las matemáticas.

El modelo metodológico MOC-ETNO, aporta al programa de etnomatemática en la construcción de caminos de diálogos interculturales, como estrategia de fortalecimiento que algunos modelos no contemplan, por ejemplo, MEDIPSA diseñado por Oliveras (1996) es fundamentado en cuestiones epistémicas, sociológicas y antropológicas contemplando la realidad como única, construida socialmente a través de la diversidad contextualizada en las distintas culturas. Esta diversidad en las distintas culturas debe ser sometida a procesos de diálogos y comparaciones como conocimiento de otras etnomatemáticas, es allí donde MOC ETNO, abre caminos para propiciar dichas reflexiones.

Del mismo modo, el método de la interpretación matemática situada (IMS) diseñado por Alberti (2007) permite de algún modo que dos investigadores distintos puedan realizar diferentes interpretaciones e identifiquen diferentes matemáticas, cuando observan la misma cultura. Esta situación no la contradice el modelo metodológico MOC - ETNO, pero a diferencia trata de hacer las interpretaciones más ligadas a la episteme del miembro de la cultura y a su realidad. Todas estas conclusiones y consideraciones evidencian la singularidad y complementariedad de MOC - ETNO dentro del programa de etnomatemática.

La implementación de cada una de las fases del modelo metodológico MOC-ETNO, diseñado como el aporte teórico de la investigación, permite recolectar la información necesaria e importante durante el proceso investigativo. El modelo se constituye en estructura referencial para no perder el horizonte de la investigación y al mismo tiempo, al utilizarlo fue posible afinarlo y reestructurarlo para su adaptación y aplicación en futuras investigaciones.

En el diseño e implementación de las tres actividades de aula en la fase cuatro del modelo, se tiene en cuenta tanto el estudio etnográfico, el diálogo comparativo con otros grupos étnicos en sus formas de medir, la participación de la comunidad y de algunos docentes de 
la institución. La interrelación de estos elementos contribuye a que los estudiantes se apropien de su proceso de formación, despierte en ellos motivación para participar activamente en la clase de matemáticas y sobre todo que de una manera significativa comprendan la matemática académica.

\section{Referencias.}

Alberti, M. (2007). Interpretación situada de una práctica artesanal (Tesis Doctoral). Universidad Autónoma de Barcelona. Barcelona, España.

Ávila, A. (2017). Elementos de la cultura cabécar relacionados con nociones matemáticas que se estudian a nivel de educación primaria en Costa Rica. Innovaciones educativas, 19(26), 21-37.

Balcázar, N., González, A., Gurrola, P., \& Moysén, C. (2013). Investigación cualitativa. Toluca, México: UAEM.

Bereday, G. (1964). Reflections on comparative methodology in education. Comparative Education, 3(3), 169-287.

Bishop, A. (1999). Enculturación matemática: la educación matemática desde una perspectiva cultural. Barcelona: Paidós.

Bishop, A. (2005). Aproximación sociocultural a la Educación Matemática. Cali, Colombia: Universidad del Valle.

Caballero, A., Manso, J., Matarranz, M., \& Valle, J. (2016). Investigación en Educación Comparada: Pistas para investigadores noveles. Revista Latinoamericana de Educación Comparada, 7(9), 39-56. Recuperado de http://www.saece.com.ar/relec/revistas/9/art3.pdf

D’Ambrosio, U. (1985). Ethnomathematics and its place in the history and pedagogy of mathematics. For the learning of Mathematics, 5(1), 44-48.

D’Ambrosio, U. (2000). Etnomatemáticas entre las tradiciones y la modernidad. México: Díaz Santos.

Fuentes, C. (2014). Algunos enfoques de investigación en Etnomatemática. Revista Latinoamericana de Etnomatemática, 7(1), 155-170.

García, Mosquera y Escobar (2018). El estudio de la medida en las prácticas socioculturales del cultivo de arroz. Saberes, Diversidad y paz. $6^{\circ}$ congreso internacional de 
Uribe, D., Acevedo, M., Rojas, O., Yojcom, D. (2020) Comparación de hallazgos matemáticos en grupos culturales diferenciados mediante el modelo metodológico MOC - ETNO. Revista Latinoamericana de Etnomatemáticas, 13(2), 45-65 DOI: 10.22267/relatem.20132.60

etnomatemática, Medellín, Colombia.

Gavarrete, Martinez y Chavarría (2018). Explorando Medidas Tradicionales de Costa Rica dentro de un proceso de Formación Docente en Enculturación matemática y Etnomatemática. Saberes, Diversidad y paz. $6^{\circ}$ congreso internacional de etnomatemática, Medellín, Colombia.

Luque, A. (2010). Una metódica para el hallazgo de las matemáticas en grupos étnicos. Omnia, 16(2) pp. 20-40.

Oliveras, M. (1996). Etnomatemáticas: formación de profesores e innovación curricular. Granada: Comares.

Rosa, M., Orey, C., \& Gavarrete, M. E. (2017). El Programa Etnomatemáticas: Perspectivas Actuales y Futuras. Revista Latinoamericana De Etnomatemática, 10(2), 69-87.

Santamaría, F. (1983). El fundamento de la metodología comparativa en educación. Educar, (3), 061-75.

Trujillo, O., Miranda, I., \& De la Hoz, E. (2018). Los sistemas de medida en la comunidad Arhuaca: su uso en distintos contextos. Revista Latinoamericana de Etnomatemática: Perspectivas Socioculturales de la Educación Matemática, 11(2), 31-51. Recuperado de http://funes.uniandes.edu.co/12919/.

UNESCO. (2012). Challenges in basic mathematics education. París. Recuperado de http://unesdoc.unesco.org/images/0019/001917/191776e.pdf.

Valverde, M., \& Espinoza, A. (2012). Etno-Matematica en indígenas Ulwas comunidad de Karawala, Región Autónoma Atlántico Sur, Nicaragua. Ciencia e Interculturalidad, 11(2), $\quad$ 70-87. Recuperado de http://www.lamjol.info/index.php/RCI/article/viewFile/960/771. 